\title{
Data reconstruction and homogenization for reducing uncertainties in high-resolution climate analysis in Alpine regions
}

\author{
Emanuele Eccel $\cdot$ Piero Cau $\cdot$ Roberto Ranzi
}

Received: 30 November 2011 / Accepted: 4 March 2012 / Published online: 28 March 2012

(C) Springer-Verlag 2012

\begin{abstract}
Analysis of climatic series needs pre-processing to attain spatial- and time-consistent homogeneity. The latter, in high-resolution investigations, can rely on the strong correlations among series, which in turn requires a strict fulfilment of the quality standard in terms of completeness. Fifty-nine daily precipitation and temperature series of 50 years from Trentino, northern Italy, were pre-processed for climatic analysis. This study describes: (1) the preliminary gap-filling protocol for daily series, based on geostatistical correlations on both horizontal and vertical domains; (2) an algorithm to reduce inhomogeneity owing to the systematic snowfall underestimation of rain gauges; and (3) the processing protocol to take into account any source of undocumented inhomogeneity in series. This was performed by application of the $t$ test and $F$-test of $\mathrm{R}$ code RHtestV2. This pre-processing shows straightforward results; correction of snowfall measurements re-evaluates attribution of patterns of altitudinal trends in time trends; homogenization increases the strength of the climatic signal and reduces the scattering of time trends, assessed over a few decades, of a factor of 2 .
\end{abstract}

E. Eccel $(\bowtie) \cdot$ P. Cau

Sustainable Agro-ecosystems and Bioresources Department, IASMA Research and Innovation Centre,

Fondazione Edmund Mach - Via E. Mach 1,

38010 San Michele all'Adige, TN, Italy

e-mail: emanuele.eccel@iasma.it

R. Ranzi

DICATA-Department of Civil Engineering, Architecture,

Land and Environment, University of Brescia,

Via Branze 43,

25123 Brescia, Italy

\section{Introduction}

Management of uncertainty is a key factor in addressing the need for confidence in the climate science as required by stakeholders (Swart et al. 2009; Ha-Duong et al. 2007; Moss and Schneider 2000). In climate projections, the peculiarities of small-scale features are, in general, overlooked by climatic investigations based on General circulation models (Timbal et al. 2009; Le Treut et al. 2008; Wilby et al. 2004). Nevertheless, even in studies based on instrumental series, the problem of the uncertainties originated by inadequate representations of the climatic status cannot be neglected. Moreover, an analysis of instrumental series is often the first step for simulations of future climate, demanding reliable instrumental series to carry out statistical downscaling.

Climatic characterization is particularly critical in geographically complex areas, like the Alpine region. To reach a satisfactory representation of the climatic conditions of mountain regions, a high number of series has to be considered. Dealing with several, long instrumental series in the same area implies a spatially coherent homogenization protocol, to avoid unlikely differences in the climatic signals that can be inferred from the analysis. On the other hand, the availability of several series in the same region offers the opportunity for using homogenization techniques based on the spatial consistency of meteorological fields like precipitation and temperature.

Either as a preliminary investigation aimed at climate projection downscaling or as a stand-alone research on climate change from instrumental records, the need is clear for a careful pre-processing of climatic series. Its goal is the homogenization of instrumental series for the removal of undesired effects (both long-term errors or simply important changes in metering practices) that can alter the assessment of the true climate signal. The knowledge of the relevance of the homogenization processing is widespread. Since the 
work by Alexandersson (1986) was published, which introduced the by-now widely used Standard Normal Homogeneity Test, several methods have been proposed to spot change points in climatic series. A review on the methods of detection of such discontinuities can be found in Reeves et al. (2007). The European Union devoted one "COST Action" to this issue (ES0601: Advances in homogenisation methods of climate series: an integrated approach (HOME) $\mathrm{http}: / /$ www.homogenisation.org/index.php). In spite of a large predominance of works on monthly series, the use of daily data is mandatory in many applications, ranging from climatology of daily extremes, to the use of daily indices (Jones et al. 1999), to the application of models for simulation of ecological, biological, agricultural systems, and others. This led a few research teams to address the problem of homogenization of daily series (Yan and Jones 2008; Della Marta 2006).

The end-product of homogenization is a time series that is suitable for climatic analysis. However, the task does not carry out corrections to systematic errors; neither does it offer tools for coping with missing values. The latter are an important source of errors, especially in precipitation series, where any null record acts toward an underestimation of the aggregated value. Hence, in order to produce discontinuityand gap-free daily series, the complementary task to homogenization is the reconstruction of missing data.

In areas where a non-negligible part of the total precipitation amount is collected by rain gauges as snow, a reliable homogenization protocol must keep into account the problem of the systematic underestimation of snowfall in the cold period of the year (Yang et al. 1994). In a mountain region, this is an outstanding issue, involving concerns about the presence of snow cover, which impacts different aspects of a mountain environment (Valt and Cianfarra 2010): water availability in the spring (Groisman et al. 1994; Cohen and Rind 1991), survival of glaciers in a warming climate (Ranzi et al. 2010), ecosystem stability (Euskirchen et al. 2007; Löffler 2007; Groisman and Davies 2001), and economical and recreational use of ski resorts (Keller et al. 2005; Haubner 2002; Hantel et al. 2000). Sevruk (1996) reports underestimation for snowfalls up to $80 \%$ on the mountains, due to the inability of rain gauges in catching satisfactorily wind-drifted snow, even if other (partial) estimates, for the instrumentation used in Italy, suggest lower corrections (Anselmo 1998). The application of a correction algorithm leads to an improved confidence in time trends of precipitation in winter, but, on the mountain area, also in the autumn and in the spring. In this paper, a 50-year (1958-2007) time series of daily minimum and maximum temperatures and precipitation collected for 59 stations in Trentino, a region of the central-eastern Italian Alps, is first presented. The criteria for the reconstruction of missing data are outlined in the following section. Then, the protocol for the selection of reference series, data homogenization, and snowfall precipitation correction are described. Results are presented in the following section, where the impact of the data correction and homogenization procedure on the identification of altitudinal and time trends is discussed.

\section{Methods}

\subsection{Geo-climatic description of the area}

Trentino is a region in the central-eastern Italian Alps (Fig. 1) covering an area of $6,212 \mathrm{~km}^{2}$. Its geography is characterized by a valley system. The largest is, by far, the flat and deep glacial valley run through by Adige River in the north to south direction. Altitude ranges between $70 \mathrm{~m}$ asl and 3,769 $\mathrm{m}$ of the Cevedale peak. The low-middle elevation climatic areas of Trentino can be ascribed to a Köppen "Cfb class" ("temperate, middle latitudes climate, with no dry season"). More elevated, mountain areas (covered by the weather station network) fall into a "Dfc" classification ("microthermal climate, humid all year round"). Trentino climate is mostly oceanic, with some areas showing features of transition to a more ContinentalAlpine climate, cooler and often drier, more typical of the inner mountain valleys. Precipitation amounts are mostly distributed over two maxima, in the autumn (main) and in the spring (secondary), although, in some mountain areas, rainfall peaks in summer (Eccel and Saibanti 2007).

\subsection{Climatic series}

Time series of temperature and precipitation were obtained from several data providers: in Trentino, mostly from Meteotrentino (the meteorological service of the Autonomous Province of Trento-PAT), others from Fondazione Edmund Mach; for areas surrounding Trentino, from ARPA Veneto (ARPAV), ARPA Lombardia (ARPAL), and from the Autonomous Province of Bolzano (PAB). Stations were selected according to their geographic position, in order to sample the region as homogeneously as possible, also considering the altitudinal displacements of sites. Of course, a selection standard was also the length of the available series, which ideally had to be long enough for a climatological study. Figure 1 shows the location of the stations whose time series were used. All series were considered for the 50-year period 1 January 1958-31 December 2007, where available. Figure 2 represents the mean annual values of both mean temperature and total precipitation (period: 1978-2007), plotted according to each station elevation. Only series having at least 25 years in the period were considered.

Because the processing of series included a homogenization trial, a few time series from the PAT database were merged, as they belonged to very close stations operating at 
Fig. 1 Coverage of the Trentino region by the selected network of stations. Round grey symbols: sites of calibration of the snowfall correction algorithm

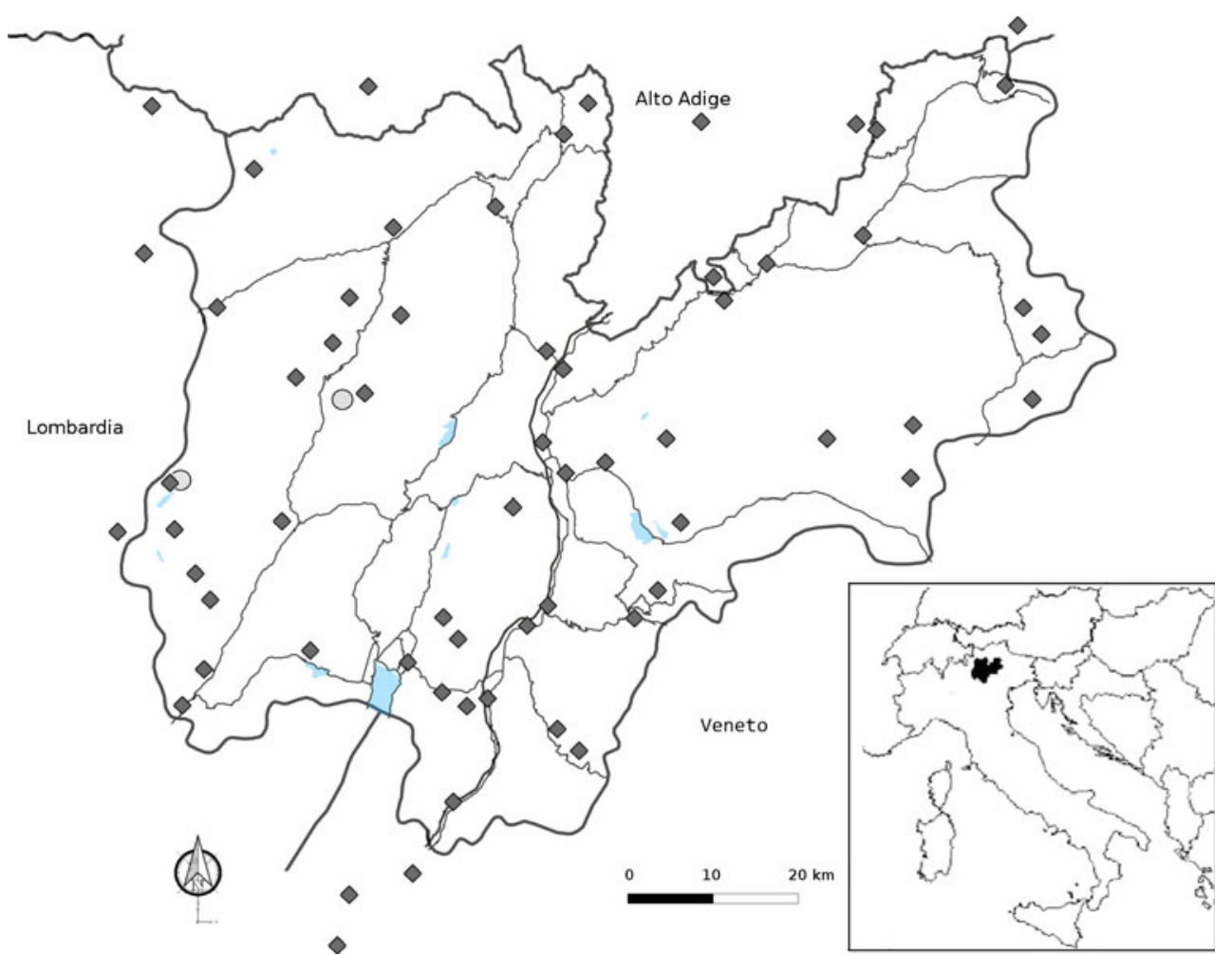

different times. The data in such series were considered as coming from the same station after relocation. As a result of these junctions, the number of time series was reduced to 59 .

\subsection{Missing daily data reconstruction}

\subsubsection{Temperature}

At the simplest level, data from the closest stations can be used to reconstruct a missing value at a given station. In operational meteorology, variants of this approach include

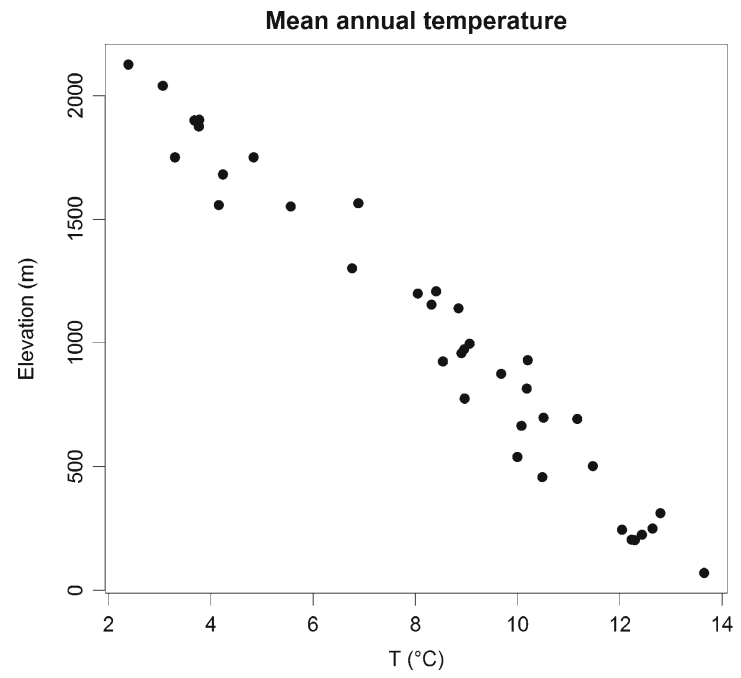

univariate methods, using data from either the closest, or the best correlated station, and statistical relationships include the application of either fixed or variable offsets (typically displaying a cyclical behavior along the year) or the use of linear regression including information on the altitudinal temperature lapse rate.

More refined approaches make use of geo-statistical, multivariate methods, by interpolation from surrounding stations. The most used methods are inverse distance weighting (IDW), splines, and kriging. The first technique attributes weights to surrounding stations as some power (an

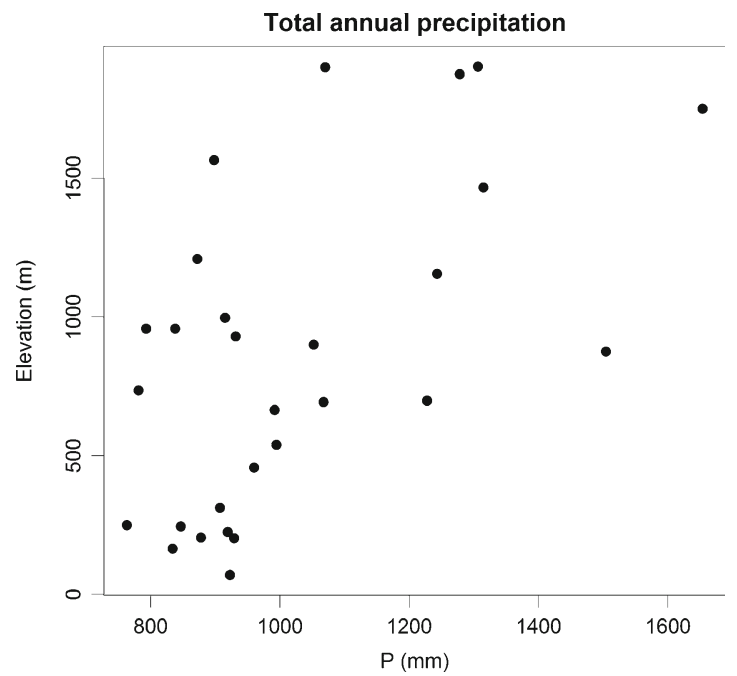

Fig. 2 Climatic values of Trentino meteorological stations as a function of station elevation, 1978-2007. Left: mean annual temperature. Right: total annual precipitation 
exponent 2 is frequently used) of their inverse distances and then reconstructs the missing value at a given station as a weighted average of measures at the other stations. All these methods can encompass algorithms for taking into account geo-morphologic features, like the station elevation, or others.

If the time series from "reference" stations were affected by lack of homogeneity, using their data would lead to poor reconstruction. For this reason, a preliminary homogenization test was performed on minimum and maximum temperatures, using a penalized maximal $F$ test (Wang 2008a, b) as implemented in the RHTestV2 package, and the related corrections were carried out (more details on this are given in section 2.4 of Methods).

Since daily data reconstruction was not the focus of the whole data processing, no multi-method comparison was carried out. Thanks to the dense data coverage in the area, an approach based on measures in the neighboring sites was applied, and the IDW method was used, including a sitespecific calibration.

IDW consists essentially of a weighted average of daily temperatures (both minimum and maximum) from other stations.

$T_{i}^{k}=\frac{\sum_{j \neq k} \omega_{j k} T_{i}^{j}}{\sum_{j \neq k} \omega_{j k}}$

where $T_{i}^{k}$ represents the reconstructed temperature at time $i$ in station $k, T_{i}^{j}$ the temperature at time $i$ in station $j$ and $w_{j k}$ represents the weight of station $j$, given its distance from station $k$. Weights are defined by an inverse power of distance only.

Before averaging, three key steps were taken. First, all temperatures were referred to the same altitude of the target station by applying a daily vertical thermal profile calculated by data from all stations. The daily change of temperature with height resulting from linear regression of temperature vs. elevation was used to account for differences in elevation in Eq. 1, where all temperature values are referred to the elevation of the candidate series. The elevation correction was different from one day to another, depending on the daily vertical profile. So the same temperature at one reference station implies generally slightly different temperatures on the target station on different days.

Even after applying this pre-processing, residual seasonality was present in temperature differences within station pairs (example in Fig. 3). To take this seasonality into account, a sinusoidal fitting was carried out to represent the residual difference and its seasonal cycle. So, the $T_{i}^{j}$ in the right hand side of Eq. 1 are temperatures projected to the same altitude of the candidate station and to which a seasonal correction $\Delta T$ has been added according to the nonlinear fitting for that day.

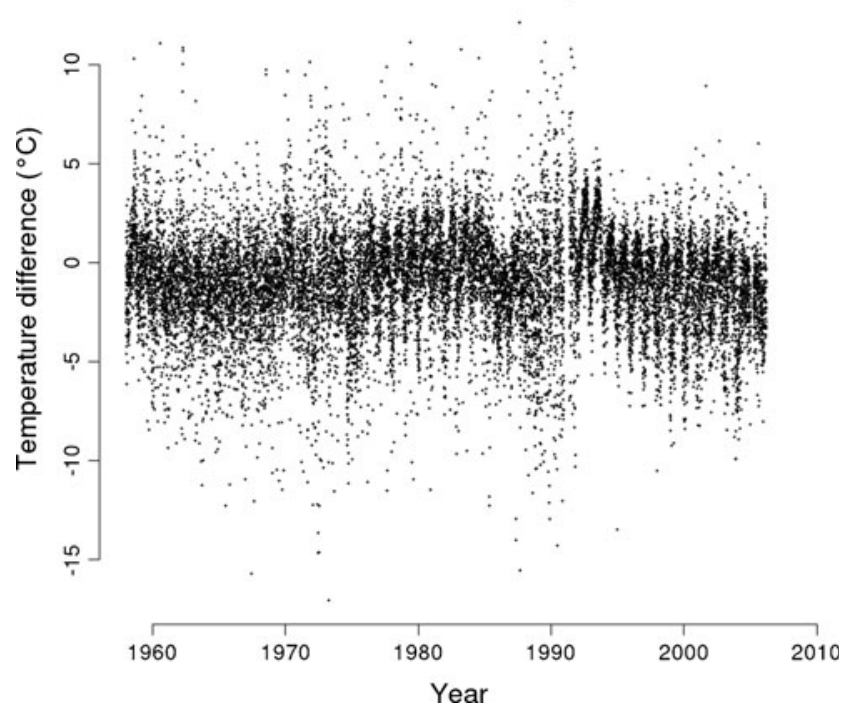

Fig. 3 Example of the temperature difference in a pair of stations after referring them to the same altitude (see text). A seasonality in residuals is detectable, even if mean difference is close to zero. A change in variance can be observed around 1990, indicative of possible inhomogeneities in one or both the series

Besides, to avoid interference of the site-specific climatic dynamics, and above all, macroscopic biases owing to differences in site elevations, the time series of temperatures were standardized before performing the actual weighted average by subtracting their mean and dividing by their standard deviation. This process was inversely applied after averaging by using the mean and standard deviation of the series being reconstructed. In this way, the reconstruction algorithm reproduces the spatially interpolated variation from the mean behavior at a site, eventually leading to the actual metric values.

The precise dependence of weights on distance affects the performance of the IDW algorithm, as in Eq. 1, the relative weights of different stations will be different with different choices of the weight formulation. A first hint should be looked for in data themselves. The most natural way is to look at correlation between stations in order to provide an estimation of the correlation radius used to choose the stations to include in the algorithm; this method was used to make sure that stations beyond this radius did not contribute to the reconstruction, as will be described later more in detail.

\subsubsection{Precipitation}

The reconstruction of missing data in daily precipitation time series is more problematic. One can still use standard interpolation techniques to calculate the missing data on a target station by using synchronous data from other stations, assuming there is a significant correlation between them. 
Sometimes, using correlation alone works better. Indeed, it has been shown that weighting the contribution from single stations on the basis of correlation alone generally outperforms reconstruction schemes based on inverse distance weighting even when different exponentials of distance are used (Ahrens 2006; Teegavarapu and Chandramouli 2005). The problem with the reconstruction of missing values of daily precipitation is that weighting methods such as IDW, as well as regression-based methods, are all affected by a systematic tendency to overestimate the number of rainy days, because of the irregular spatial and temporal pattern of precipitation. Besides, rainfall probability distributions are not preserved (Simolo et al. 2010); this problem also affects more advanced statistical techniques such as those based on neural networks.

These two problems represent a serious difficulty, particularly when the reconstructed time series are used to perform climate analyses that make use of synthetic weather generators, which strongly depend on a proper knowledge of rainy days and the probability distributions of rain amounts.

To confront these problems, Karl et al. (1995) devised an approach based on fitting a two-parameter Gamma distribution to the data of each station, deciding which days are rainy and using random numbers to determine the amount of precipitation on the rainy days. To decide if a day is rainy, another random number is used, whose distribution is the empirical probability of rainy days on that day of the year. This approach is very effective in preserving both the number of rainy days and the statistical distribution of rainfall, though it randomly locates precipitation events, making it of little use in the reconstruction of daily missing data. To improve on this approach, Simolo et al. (2010) used the same ideas of Karl et al. (1995) while introducing back the use of specific weighting average of probabilities in surrounding stations (where weights are functions of distance, elevation difference, and relative angular distribution) to determine if a day is rainy on the target station, thus removing the problem of random location of precipitation events. Once a day is known to be rainy, the amount of precipitation is calculated via a multi-linear regression.

We used the same approach of Simolo et al. (2010). A pre-homogenization was carried out also for precipitation series.

\subsection{Monthly series homogenization protocol}

The general scheme adopted for monthly series homogenization is represented in Fig. 4. The algorithm includes a first-level, "auto-homogenization", performed on single time series, and a second-level homogenization, carried out with the help of a reference series considered as homogeneous. Both processing steps were carried out by the $\mathrm{R}$

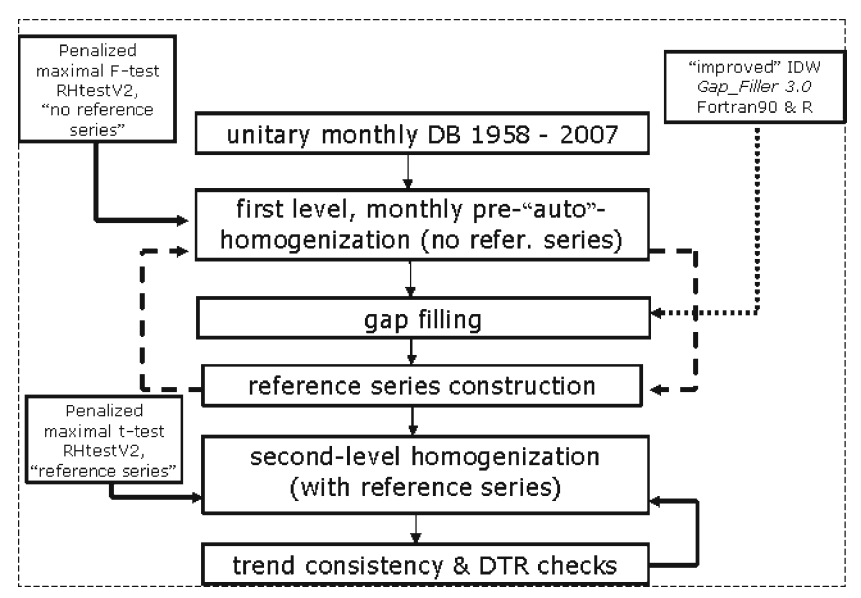

Fig. 4 Flow chart of the homogenization protocol

code "RHtestV2" (Wang and Yang 2007; Wang 2008a, b). The package, developed for the open-source $\mathrm{R}$ code ( $\mathrm{R}$ Developing Core Team 2011), performs homogenization of climatic series both with and without homogenous reference series ( $t$ test and $F$ test, respectively). The homogenization can be carried out on both temperature (minimum and maximum) and precipitation, the latter needing a previous log-transformation.

A preliminary homogenization test was performed using a penalized maximal $F$ test, and the related corrections were carried out. This test does not use reference series and, allowing for linear trends to be present in the series, detects mean shifts with no change in trend. Whenever available, metadata were used by testing the series under the assumption that discontinuities occur in correspondence with documented changes.

After filling the gaps in all daily series, for each station a (presumed) homogeneous reference series was built, necessary for the second-level homogenization process. The reference series were built by a non-weighted average of the five closest neighbouring stations. In the case of temperature, this averaging was subjected to the same preprocessing described in section 2.3.1 for the reconstruction of missing data, to take into account the difference in altitude between stations and the presence of seasonality in this difference. This averaging is meant to provide a reliable representation of the general climatic features in the area around each station and has the advantage of smoothing the effects of the presence of inhomogeneities that had not been corrected in the preliminary homogenization. The attribution of equal weights for every site (arithmetic mean) avoids the enhancement of inhomogeneities that could affect the composite, reference series when present in the series more highly correlated with the candidate one. In a weighted mean, inhomogeneities of such series would be magnified by high weights. 
A reference series for each station allows one to discriminate between changes in the time series that are also present in the reference series - therefore, likely due to actual discontinuities in the time series-from discontinuities that are not present in the reference series, suggesting the presence of inhomogeneities of probably non-climatic origin in the time series under test.

The $\mathrm{R}$ code RHtestV2 prompts the user a series of change points, found after the set up of a significance level ( $5 \%$ as default). The user, helped by a graphical representation of the difference with the reference series, can accept, reject, or shift such points in time; more points can be added as well, resulting from different information sources (station metadata). When possible, some change points were forced to assume the values of the discontinuities recorded in metadata files. For temperature, some forcing was done also to match the change points between minimum and maximum series. When metadata were not available, a conservative approach was used, by accepting only potential discontinuities with large statistical significance. After every change in the change-point list, the algorithm computes again the statistical significance of the new setup. The procedure was carried out iteratively, until a satisfactory interpretation of the discontinuity points was attained. The result is a homogenized series, obtained from the raw one by introducing a series of positive or negative steps (both in temperature and in the logarithm of rainfall depth). Series are always homogenized to their latest period, namely adjustments are always zero at their final end.

Given that the definition of the change points is, to some extent, prone to arbitrariness, owing to the subjective choices of the user, direct results of the second-level homogenization process have undergone two further inspections. The first was a comparison of the time trends of each station with the others. Time trends were calculated for two periods (1958-2007 and 1978-2007), excluding series with less than 40 and 25 years, respectively. Series having trends outside the range "mean (of time trends) $\pm \sigma$ " $(\sigma=$ standard deviation) were critically inspected and, in case, put through a new homogenization trial.

The other check consisted in finding discrepancies between homogeneity in series of minimum and maximum temperature (no equivalent method was enforceable for precipitation series). In fact, RHtestV2 calculates homogeneity adjustments on single-variable series. So, despite the efforts done by matching change points in minimum and maximum series, some inhomogeneity was still detectable by comparing single daily thermal range trends ((DTR) monthly differences between means of daily maxima and minima) with a curve averaged over all series, and, above all, by superimposing the "step curves" of the minima and maxima adjustments to every DTR series (Fig. 5). Also in those cases, the homogenization adjustment trials underwent revision.

Finally, the correction "steps", as offsets of either temperature or log-precipitation, were applied to the daily series, conventionally from the day 1 of each month of inhomogeneity detection. In the precipitation series, no alteration was brought in the number of rainy days, so the change in precipitation rate was applied to the existing precipitation days.

\subsection{Correction for snowfall underestimation}

The problem of the systematic underestimation of snowfall, due to the limited ability of rain gauges to catch snow, cannot be directly tackled by a homogenization algorithm based on the search of point discontinuities. Nevertheless, it is a fact that a temperature increase along decades decreases the fraction of snow precipitation over the total, leading to a progressive decrease of the underestimation of precipitation in the snowy period of the year.

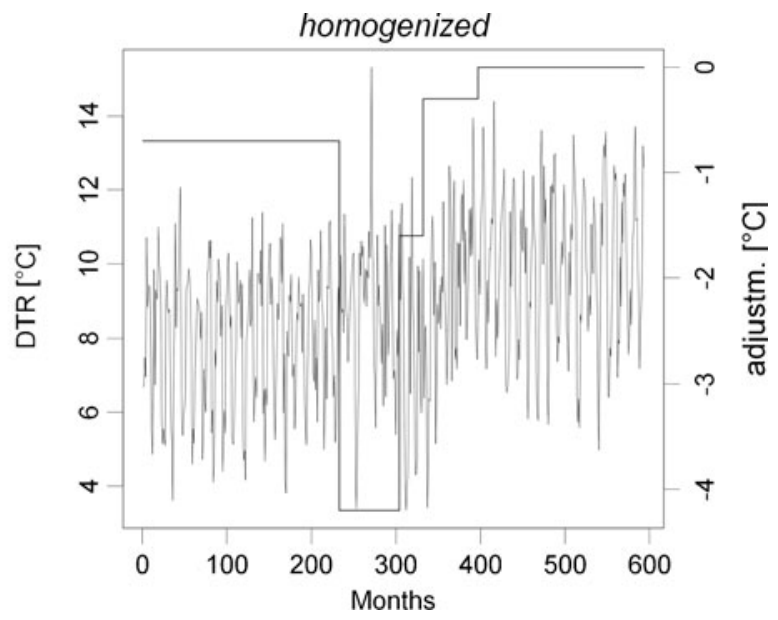

Fig. 5 Example of detection of a probable wrong attribution of a change point in daily temperature range (DTR contraction after application of correction). Continuous line: DTR. Broken line: adjustment steps applied after homogenization 
The problem of systematic underestimation of snow precipitation by rain gauge measurements was investigated by several authors, as Sevruk (1992), but corrections by hydrometeorological services, although recommended by international agencies, are not frequent (WMO 2010). An example of correction of precipitation is given by the Swiss Hydrological Atlas, where a map of mean annual precipitation depths is reported with corrections depending on topography and meteorological variables, as wind speed, precipitation, and temperature. But, several climatologies of Alpine precipitation (see e.g., Auer et al. 2007 and Brunetti et al. 2000 for the Italian Alpine precipitation) do not apply systematic snow precipitation correction.

A sound correction of the underestimation needs the simultaneous knowledge of, at least, temperature and snowfall, measured as melted snow by rain gauges. Mean monthly temperature offers a poor detail on the nature of single events; then, the availability of gap-free daily series can be considered as mandatory for a rigorous correction of the underestimation of precipitation during snow events. Given the predominance of rain gauges on thermometers in meteorological networks of the past, not for all sites is it possible to attribute a measured temperature to any precipitation event, making a reliable discrimination between rainfall and snowfall a difficult task. However, during significant winter precipitation events, in general, air flow dynamics is not hampered by catabatic flow mechanisms or by strong microclimatic features. In such conditions, thermal gradient close to the ground is well approximated by the mean daily thermal gradient calculated by all the available stations. Therefore, in all sites where only precipitation measures were available, the reference daily temperature was assessed by linear regression on station elevation, calculated by the temperatures measured at all the available thermometers in the network.

The correction algorithm is simple, and suggested by the work by Ranzi et al. (1999), who estimated, for a full-snowy precipitation, underestimation by $30 \%$ to $70 \%$. Of course, the bias is strongly dependent on the wind velocity during the snowfall. The latter is not assessed in the present climatic analysis, owing to the low number of weather stations equipped with anemometers, especially in the past, and particularly at mountain sites; hence, a general rule, based on temperature only, had to be formulated.

In our analysis, the correction of solid precipitation measurements is based on experimental data collected at the meteorological station of Malga Bissina, located at an altitude of 1,792 masl (see Fig. 1) close to a snowfield (1,780 masl), where snow depth and fresh fallen snow depth and density are measured as well. The observation period spans the 1989-2005 years. Precipitation measured by a heated tipping-bucket rain gauge with $0.1 \mathrm{~m}$ diameter and without wind shields was accumulated in the $24 \mathrm{~h}$ until 9:00
AM of each day, providing the uncorrected precipitation data, $P$. At the same time, daily minimum and maximum air temperature were recorded. As the heating system may have taken some time to melt the snow fallen in the gauge, in case no precipitation had been measured in the following days in two nearby stations (La Rocca and Tione), the precipitation measured at the reference station in those days was assumed to be snowmelt and was added to the $P$ recorded in the measurement day. For those days when the snow records reported rain on snow, data were considered separately. Fresh fallen snow depth $h_{\mathrm{n}}$ (centimeters) and density $\rho$ (kilograms per cubic meter) were conducted in the morning of each day with snowfall, generally between 7:30 and 8:00 AM. Precipitation measurements, $P$, were compared with fresh fallen snow water equivalent, $P_{\mathrm{n}}=h_{\mathrm{n}} \rho / 100$.

As shown in Fig. 6, where all the data for the 1989-1995 period are plotted, the underestimation of snow measurement is systematic. Only 15 data out of 107 indicate values of rain gauge precipitation slightly higher than snowfall water equivalent. On average, the 107 snowfall events contributed with $2,887 \mathrm{~mm}$ of water equivalent and the heated rain gauge measured only $1,633 \mathrm{~mm}$, with an underestimation of $43 \%$ of the 'true values' $P_{\mathrm{c}}$. In order to implement a simple precipitation correction using easily available meteorological measurements, as mean daily temperature, the snow data of Malga Bissina and of Pinzolo Prà Rodont $(1,530 \mathrm{masl})$ were analyzed to identify the influence of temperature on the solid or liquid phase of precipitation. Three categories of events were selected: (a) snow precipitation only, (b) mixed rain and snow, and (c) rain precipitation only. Corresponding measured precipitation in the nearby rain gauge were plotted according to the measured mean daily air temperature in Fig. 7. Less than $30 \%$ of the 14 rainy precipitation events occurred with daily mean temperature less than $0{ }^{\circ} \mathrm{C}$, mixed rain and snow fell at temperatures between $-2{ }^{\circ} \mathrm{C}$ and $+2.5{ }^{\circ} \mathrm{C}$, while $30 \%$ of

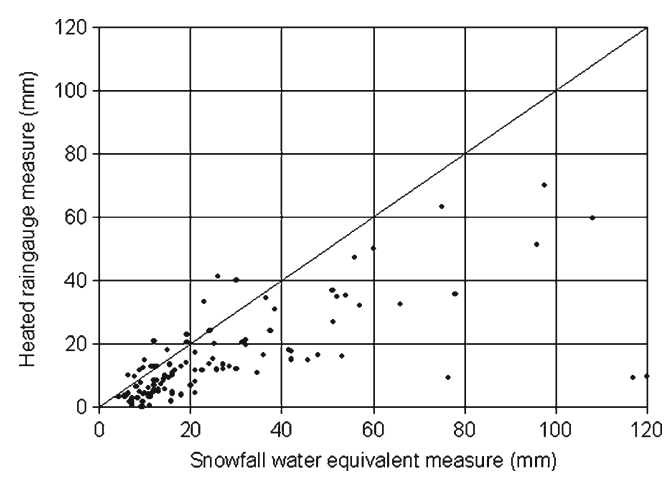

Fig. 6 Comparison of fresh fallen snow water equivalent at Malga Bissina snowfield (1,780 masl) and contemporary daily precipitation measurements at the nearby rain gauge station $(1,792$ masl) in days with snowfall 


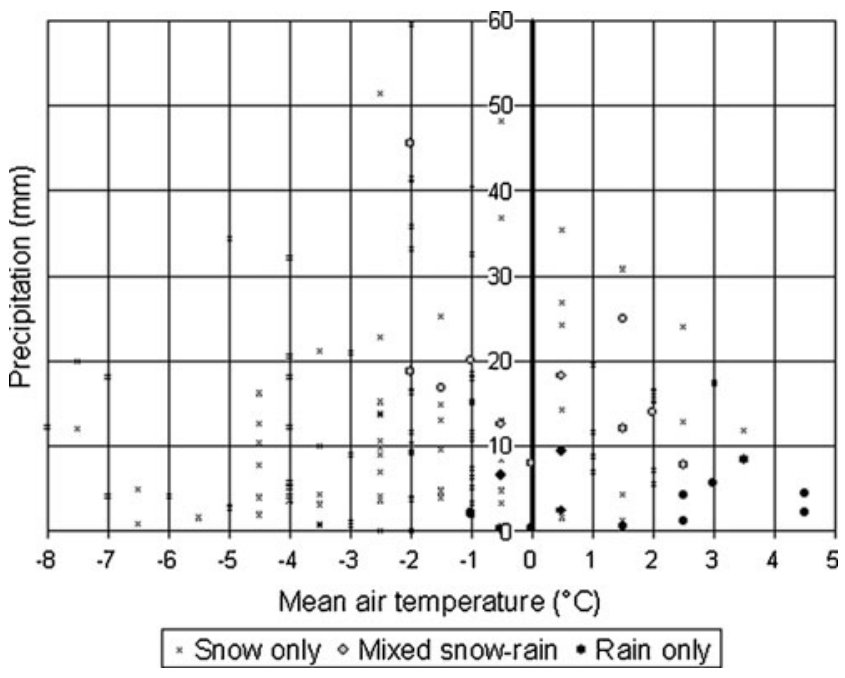

Fig. 7 Mean daily air temperature and phase of precipitation. Data from Malga Bissina and Pinzolo Prà Rodont snow fields in 1989 to 1995 years

the snow-only precipitation fell with air temperature above $0{ }^{\circ} \mathrm{C}$ and only $3 \%$ with temperature above $2{ }^{\circ} \mathrm{C}$.

Based on these experimental data, the snow-correction algorithm applied a linear augmentation of the daily precipitation $P$ according to the following equations:

$P_{\mathrm{c}}=\left(1+\alpha_{\mathrm{s}} C_{\mathrm{s}}\right) P$

where $P_{\mathrm{c}}=$ corrected daily precipitation height, $C_{\mathrm{s}}=$ maximum snowfall increase correction (set to 0.5 to minimize the spread of corrected data vs. measured water equivalent), and $\alpha_{\mathrm{s}}$ is the correction coefficient to apply to $C_{\mathrm{s}}$, according to:

$$
\alpha_{\mathrm{s}}=1 \quad \text { for } T_{\mathrm{m}} \leq T_{1}
$$

$\alpha_{\mathrm{s}}=\left(T_{\mathrm{h}}-T_{\mathrm{m}}\right) /\left(T_{\mathrm{h}}-T_{\mathrm{l}}\right) \quad$ for $T_{\mathrm{l}}<T_{\mathrm{m}}<T_{\mathrm{h}}$

$$
\alpha_{\mathrm{s}}=0 \quad \text { for } T_{\mathrm{m}} \geq T_{\mathrm{h}}
$$

with $T_{\mathrm{m}}=$ mean daily temperature (average between maximum and minimum), $T_{1}=$ lower threshold for correction $\left(0^{\circ} \mathrm{C}\right)$, $T_{\mathrm{h}}=$ higher threshold for correction $\left(2^{\circ} \mathrm{C}\right)$.

\section{Results and discussion}

\subsection{Missing data reconstruction}

\subsubsection{Temperature}

After careful analysis of our network, we chose to use weights in Eq. 1 increasingly depending on distance as station relative distances decrease, as explained in the section 2. An inverse distance weight was chosen in the form:

$\omega_{j k}=\frac{K}{K+d_{j k}^{2}}$

with $K=100$. As Fig. 8 shows, this weight decreases to about 0.4 within $10 \mathrm{~km}$ and to 0.2 within $20 \mathrm{~km}$, not as fast as $d^{-2}$ would do. A distance of about $10 \mathrm{~km}$ is in the range of the typical distance between a station and the neighboring ones in our network. Stations that are as far as $30 \mathrm{~km}$ or more are given a comparatively small weight and do not contribute significantly to the data reconstruction (Eq. 1). The plot also shows an inverse distance decay (thin line) for comparison. We found a peak of the relative distance between stations around $40 \mathrm{~km}$, with most stations within the first $20 \mathrm{~km}$ from each others. We concluded that $20 \mathrm{~km}$ is a fit distance for providing most of the information used in the reconstruction algorithm.

The overall root-mean-square error (RMSE) for temperature reconstruction averages in absolute value about $0.5{ }^{\circ} \mathrm{C}$ for monthly means, hence, a negligible average bias is attained with this technique. RMSE was about $0.8^{\circ} \mathrm{C}$ lower than with a method based on the closest neighbor (tested as a benchmark).

\subsubsection{Precipitation}

We used the approach of Simolo et al. (2010) to fill missing records of daily precipitation. This involved the use of running windows of varying width over which the fitting of a gamma distribution and the multi-linear regression were performed. Because of a higher fraction of missing data, in our case, the running windows had to be wider so to include a comparable number of data (150 or more). While this might have degraded the reconstruction, particularly by including data from too different periods of the year, the performance of the method turned out to be quite satisfactory.

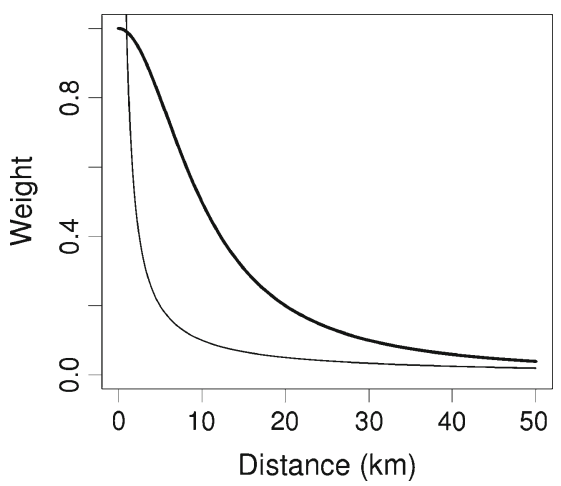

Fig. 8 Plot of the inverse distance weight used in our investigation (thick line). As a reference, a weight of the form $1 /$ distance is also shown (thin line) 
To evaluate the performance of rainfall reconstruction, we first validated the method with reference to the correct identification of dry and wet days, by a leave-one-out approach. The good functioning of the method is evident in assessing the number of rainy days. The fraction of hits is in the range 0.7-0.9, which is in line with the results obtained by Simolo et al. (2010), particularly when considering the higher percentage of missing data in our time series. Few stations had a higher fraction of misses, mainly because either they were at the edge of the network (weights are very sensitive to the network geometry) or had problematic precipitation records.

To assess the performance of the reconstruction of rainfall amounts in wet days, in Fig. 9, we show the RMSE, mean absolute error, and mean error (ME). While the RMSE is about $4 \mathrm{~mm}$, which can be considered quite acceptable and in line with similar results obtained by the authors of the method, the ME clearly shows the absence of biases in the reconstruction of rainfall amounts.

\subsection{Series homogenization}

A summary comparison of the results of the homogenization is given in Table 1 (precipitation) and in Table 2 (temperature). The strong reduction in the scattering of time trend values is evident, with singular, remarkable cases. In general, standard deviations of trends within the groups are strongly reduced. In particular for temperature, after homogenization, they are roughly halved on average. Effects on the number of series significantly increasing or decreasing are opposites, according to the strength of the climatic signal (Tables 1 and 2). When this signal is strong (as for maximum temperature), the improvement of the series homogeneity increases the number of those having a significant temperature rise, reaching $100 \%$ for the spring, the summer, and the annual aggregations. On the contrary, when the climatic signal is weaker (as for minimum temperature and precipitation), the general trend is almost flat, and most time

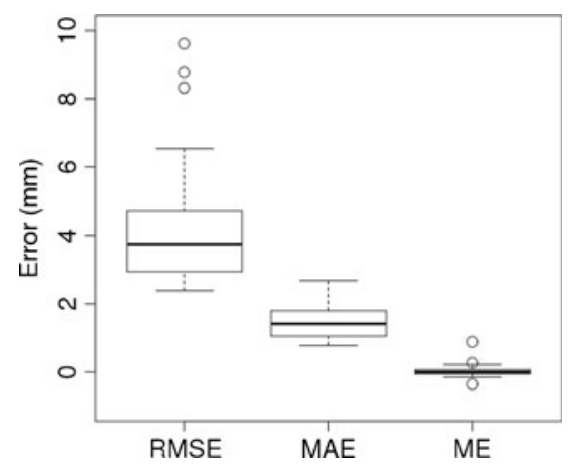

Fig. 9 Boxplot of RMSE, mean absolute error, and mean error of the reconstruction of daily precipitation. Bold lines: medians. Boxes: interquartile ranges (IQR). Whiskers: $\max$ and min values not exceeding each 1.5 IQR. Circles: outer values trends are non-significant. In this case, homogenization smoothes out those non-climatic features of the time series that had biased one or both ends of the series, leading to artificial, sometimes significant, departures from the nonsignificant mean benchmark. As a result, the number of series with significant time trends decreases after homogenization. The frequency curves of the time trends of all stations, before and after homogenization (Fig. 10), confirm the important reduction in trend uncertainty for the whole region. Time trend values are very similar to those obtained for Switzerland, for a period almost identical to ours, by Ceppi et al. (2012).

Very few homogenized temperature series display negative time trends, whereas this occurs for a number of raw series. However, in the autumn, the average trend of homogenized series is markedly lower than in the other seasons, being even negative for minima. The autumn trend of maxima is low but still significant in $2 / 3$ of the series; hence, most mean temperatures have a positive trend. The strongest increase, both for minima and maxima, was experienced for summer temperatures.

For all seasons, the thermal increase of the second half of the century can be ascribed to a larger extent to maximum temperatures. The same behavior was found in other works: In the Italian Alps (Ciccarelli et al. 2008), as well as in other Italian regions (Toreti and Desiato 2008), even if there is evidence of opposite results in other areas, for example in Umbria (central Italy - Vergni and Todisco 2011), or in Switzerland (Beniston et al. 1994 - series analyzed in this work end in the early 1990s). This peculiarity could be explained by a general decrease in cloudiness, as well as in atmospheric moisture, which indeed seems to be a feature of the last decades in the southeastern Alpine region (Brunetti et al. 2009). However, this is not the case for southwestern Alps, where maximum temperatures were found to increase more than minima (Ciccarelli et al. 2008), even if at a minor extent, if compared with the southeastern Alpine region.

For precipitation, after application of the algorithm of correction of snowfall underestimation, winter is the only season for which a few time series (eight) show a significant decrease. This number is reduced to six for annual series. For the other seasons, trends are negligible, and the number of series with a significant signal is very low (null for autumn). A work by Brugnara et al. (2011), carried out on a region that encompasses Trentino, and extends north to the more Alpine area of the central-eastern Italian Alps (Alto Adige/South Tyrol), allows interesting comparisons for any aggregation time period. For the two 50- and 30-year periods considered in our work, there is a good agreement when the regional average series is considered. Also in that case, the only season with a significant negative trend is the winter, while, in general, shorter period oscillations lower the significance of the change. 
Table 1 Fifty-year time trends in precipitation for all series (millimeters per year): comparison between raw and homogenized series, both with and without snow correction

Mean of time trends, standard deviations, and percent of series with significant trend (Pearson's $p<0.05$ )

\begin{tabular}{|c|c|c|c|c|c|c|c|c|c|}
\hline & \multicolumn{3}{|l|}{ Raw } & \multicolumn{3}{|c|}{ Homogenized, no snow corr. } & \multicolumn{3}{|c|}{ Homogenized, snow corr. } \\
\hline & Mean & $\mathrm{SD}$ & $\begin{array}{l}\text { \% Sign. } \\
\text { series }\end{array}$ & Mean & $\mathrm{SD}$ & $\begin{array}{l}\text { \% Sign. } \\
\text { series }\end{array}$ & Mean & SD & $\begin{array}{l}\text { \% Sign. } \\
\text { series }\end{array}$ \\
\hline Year & -0.594 & 3.327 & 22.20 & -1.171 & 2.111 & 5.56 & -1.941 & 2.034 & 16.67 \\
\hline Winter & -0.931 & 0.622 & 13.89 & -1.047 & 0.623 & 16.67 & -1.546 & 0.791 & 22.22 \\
\hline Spring & -0.117 & 0.913 & 2.70 & -0.213 & 0.679 & 2.70 & -0.279 & 0.750 & 2.70 \\
\hline Summer & 0.186 & 1.037 & 10.81 & -0.092 & 0.824 & 2.70 & -0.102 & 0.821 & 2.70 \\
\hline Autumn & 0.374 & 1.334 & 2.78 & 0.324 & 1.006 & 0.00 & 0.161 & 0.942 & 0.00 \\
\hline
\end{tabular}

In an Alpine area, it is of a certain interest to look for altitudinal patterns in climatic features. For this reason, values of time trends of precipitation were put into relation with station elevations (Fig. 11), to detect any pattern. Graphs represent the trends with elevation of annual series, both before and after homogenization, for two periods: 1958-2007 and 1978-2007. No snowfall correction algorithm was applied at this stage. The strong contraction in the confidence interval $(0.05 \leq p \leq 0.95)$ after gap filling and homogenization is clear. In some cases, the altitudinal trend of the rate of change with time is significant.

Precipitation time trends for homogenized series, not corrected for snowfall, show a pattern with station elevation, displaying an often lower decrease along the period for mountain sites, often reversing in sign for station heights above, roughly, 1,000-1,200 masl in 50-year trends; a less clear threshold can be inferred in 30-year trends. In general, homogenization makes the pattern clearer, albeit trends with

Table 2 Fifty-year time trends (degree Celsius per decade) in minimum temperature and maximum temperature: comparison between raw and homogenized series

\begin{tabular}{|c|c|c|c|c|c|c|}
\hline & \multicolumn{3}{|l|}{ Raw } & \multicolumn{3}{|c|}{ Homogenized } \\
\hline & Mean & SD & $\begin{array}{l}\text { \% Sign. } \\
\text { series }\end{array}$ & Mean & SD & $\begin{array}{l}\% \text { Sign } \\
\text { series }\end{array}$ \\
\hline \multicolumn{7}{|c|}{ Minimum temperature } \\
\hline Year & 0.078 & 0.245 & 37.50 & 0.094 & 0.089 & 37.50 \\
\hline Winter & 0.113 & 0.273 & 21.74 & 0.112 & 0.144 & 13.04 \\
\hline Spring & 0.115 & 0.201 & 33.33 & 0.118 & 0.127 & 20.83 \\
\hline Summer & 0.147 & 0.264 & 45.83 & 0.178 & 0.113 & 54.17 \\
\hline Autumn & -0.051 & 0.270 & 33.33 & -0.018 & 0.114 & 4.17 \\
\hline \multicolumn{7}{|c|}{ Maximum temperature } \\
\hline Year & 0.558 & 0.269 & 75.00 & 0.576 & 0.110 & 100.00 \\
\hline Winter & 0.602 & 0.359 & 78.26 & 0.630 & 0.209 & 95.65 \\
\hline Spring & 0.689 & 0.268 & 83.33 & 0.688 & 0.146 & 100.00 \\
\hline Summer & 0.700 & 0.319 & 83.33 & 0.727 & 0.166 & 100.00 \\
\hline Autumn & 0.238 & 0.321 & 37.50 & 0.263 & 0.157 & 66.67 \\
\hline
\end{tabular}

Mean of time trends, standard deviations, and percent of series with significant trend (Pearson's $p<0.05$ ) elevation are significant only for the 50-year period and neither in winter, nor in summer.

Corrections done on the original precipitation series are, in general, less important than for temperatures, probably due to the minor effect of a rain gauge move, or of the junction of two series, compared with the impacts on temperature measurements. In general, the reduction in the scattering of trends is smaller than in the case of temperatures. In some cases, the pattern with height was significant already in the raw series.

\subsection{Correction for snowfall underestimation}

Given the progressive temperature increase in the last decades, some effect of the snowfall correction was expected on time trends, at least for stations at higher elevations, where the snow fraction of precipitation is relatively high. The action is expected to strengthen negative winter trends, owing to the effect of correction of the underestimation of snowfall in the coldest seasons of the series, more frequent in their early period. Table 1 reports on the impact of the snow correction algorithm for snowfall underestimation on the time trends. There is some measurable effect, above all, on winter trends, as expected, which are passed on to the yearly values. The increase in time trends also increases the number of series with significant time trends.

As pointed out, precipitation time trends for homogenized series, uncorrected for snowfall, showed a pattern with station elevations, where the decrease was often lower for mountain sites, in many cases reversing in sign (Fig. 12). The trend with height was highly significant $(p=0.003$ ) for raw data. After the application of snowfall underestimation correction, this trend becomes non-significant at $p=0.05$. Actually, precipitation recovery produced by the correction in the cold season is inversely proportional to temperature; snowfalls are more frequent at higher elevations, accounting for a larger fraction of the total precipitation amount than at lower-elevation stations. Being the correction applied more often in the earlier, colder period of the series, time trends for the more elevated stations are shifted toward more negative (or less positive) rates, leading to poorly significant trends with elevation, with the only exception of spring trends. 

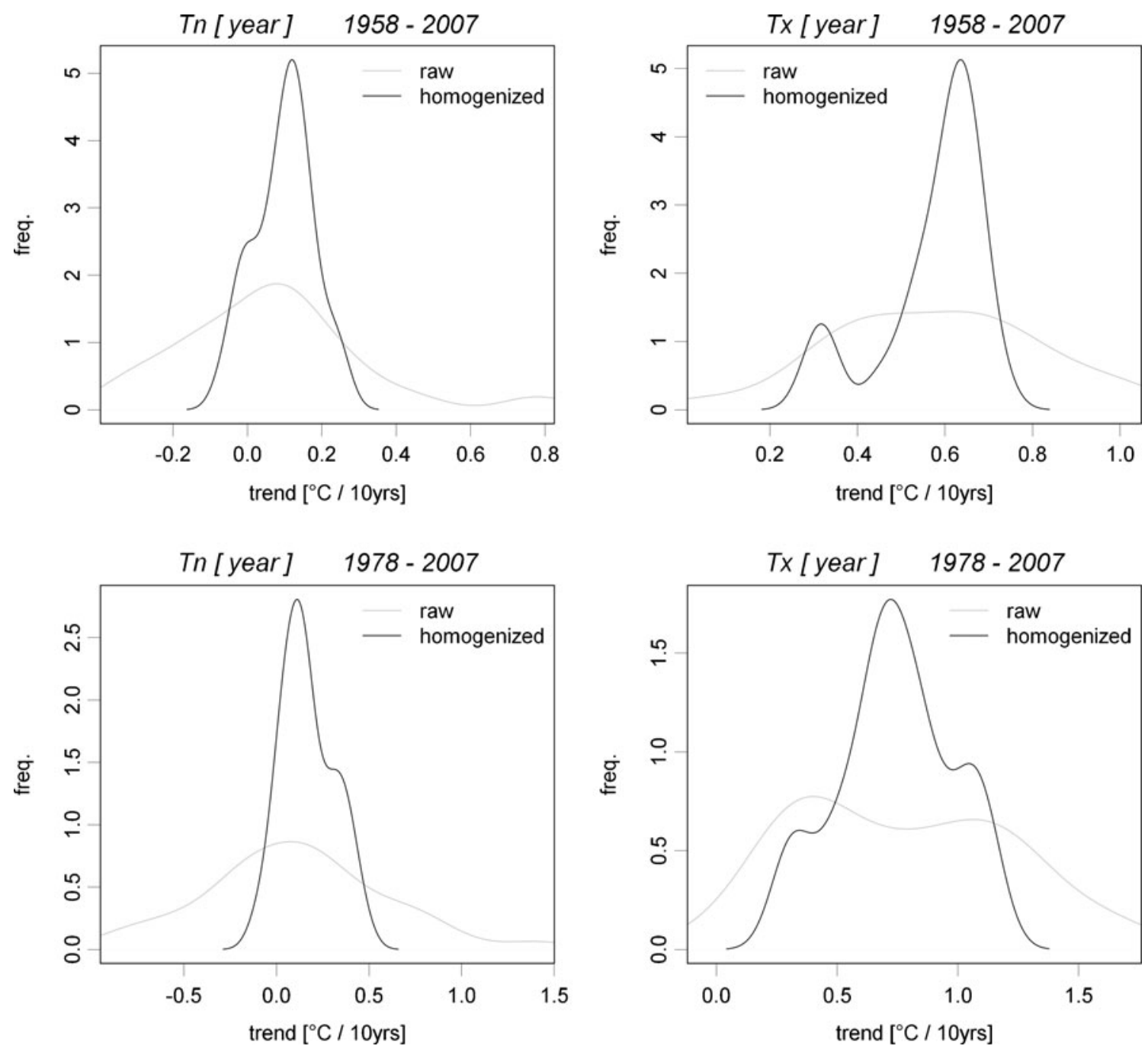

Fig. 10 Frequency curves of temperature time trends: raw vs. homogenized series. Upper panels: 1958-2007. Lower panels: 1978-2007 Left panels: minimum temperature. Right panels: maximum temperature
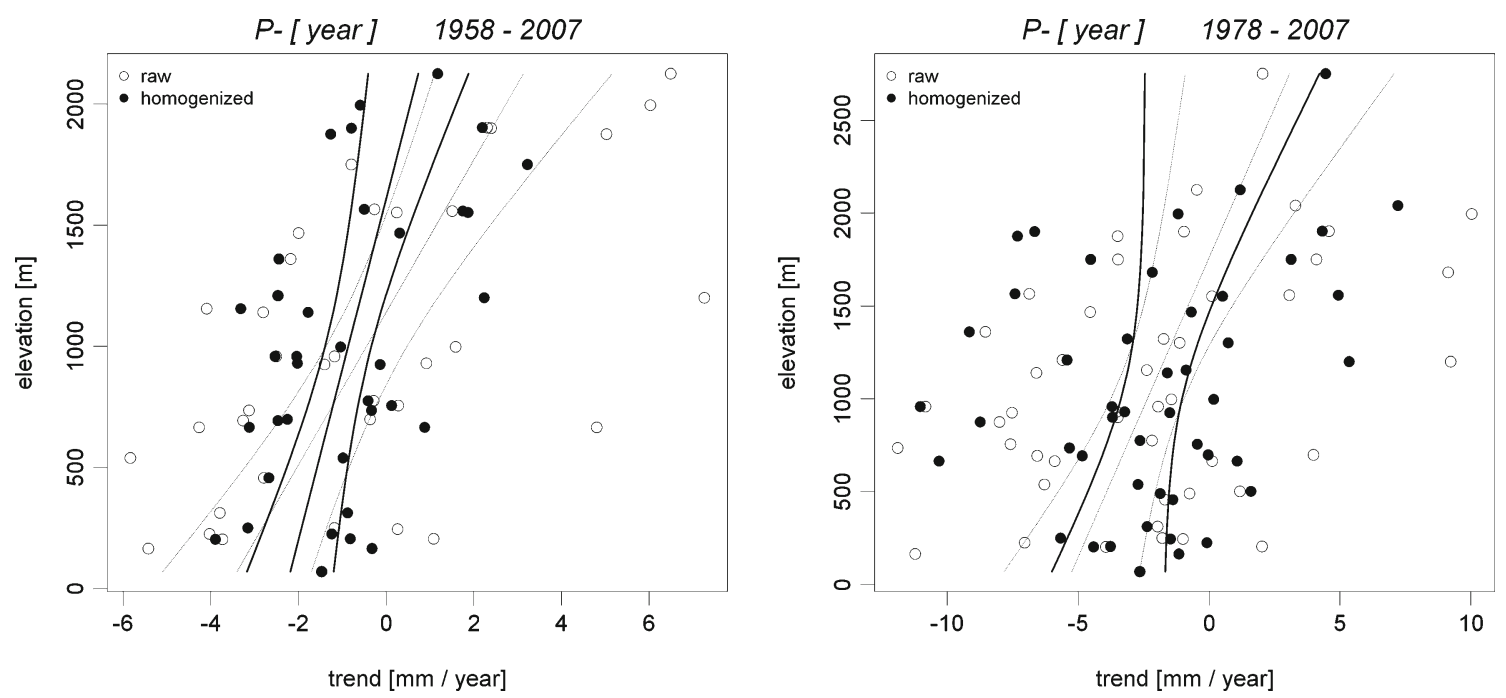

Fig. 11 Time trends of mean annual precipitation depths vs. station elevation: comparison between raw and homogenized series. Regression lines are plotted only if significant at $5 \%$ level. Confidence intervals (5\%-95\%) are plotted. Left: 1958-2007. Right: 1978-2007 

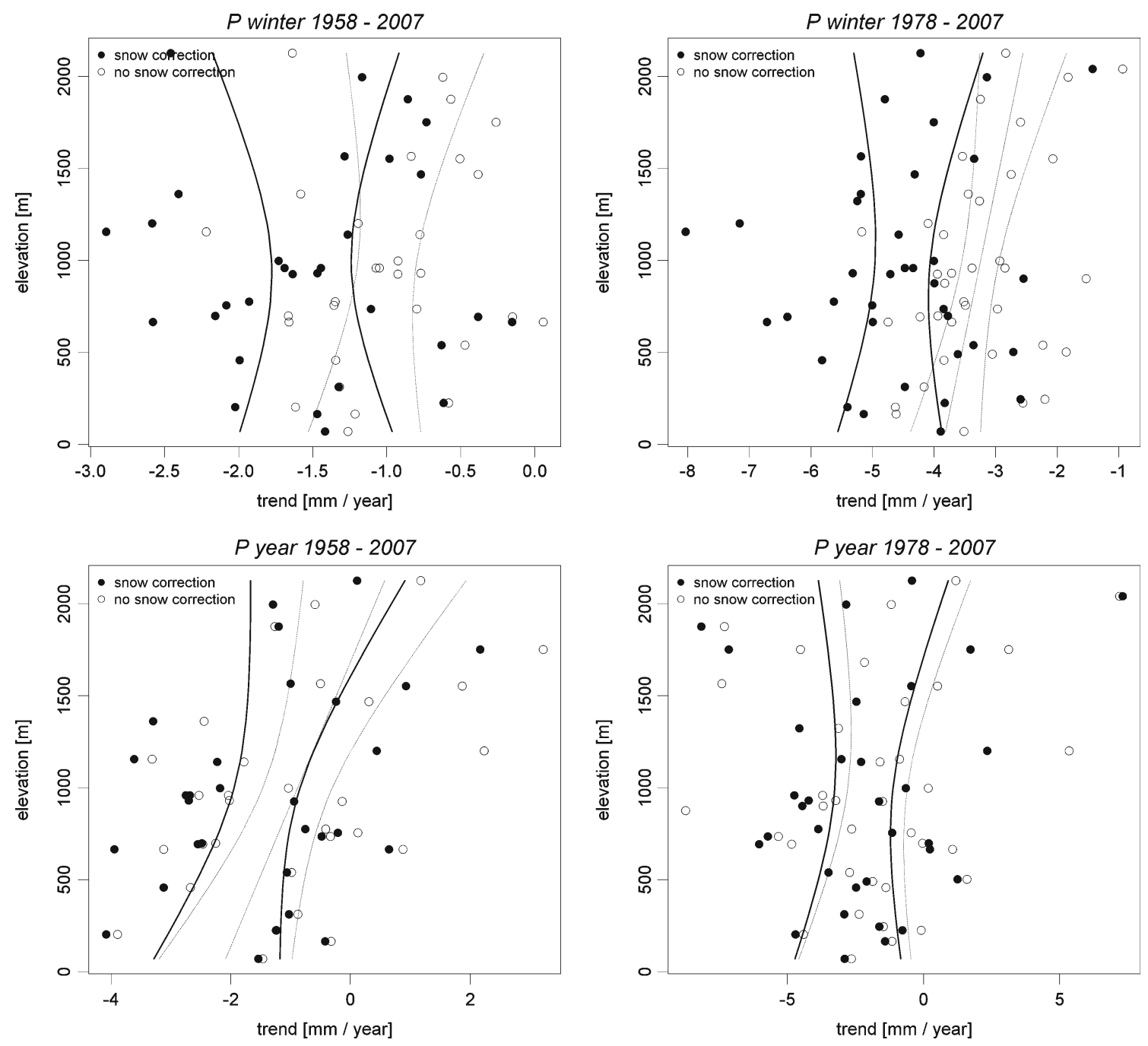

Fig. 12 Time trends of mean winter (upper panels) and annual (lower panels) precipitation heights vs. station elevation: effects of snowfall correction. Regression lines are plotted only if significant at $5 \%$ level.

Confidence intervals (5\%-95\%) are plotted. Left: 1958-2007. Right: 1978-2007

\section{Conclusions}

This work illustrates the positive action of a pre-processing of temperature and precipitation series-consisting in both missing value reconstruction and homogenization-before their climatic analysis. While temperature gap-filling techniques, based on geostatistical correlations on both horizontal and vertical domains, give satisfactory straightforward results, for precipitation the definition of a missing day as rainy or not, requires a more cautious approach.

The improvement in observed series is evident and helps to recognize climatic features that might remain concealed by the "noise" superimposed to series owing to either shifts of the meteorological stations, or to changes in the metering protocol, or to prolonged instrumental errors, including systematic snowfall underestimation. Series homogenization can lead to opposite effects on the significance of a climatic

signal. If the latter is weak, single significant time trends in series may be due to important inhomogeneities, and their removal may have as a consequence the loss of significance of the changes. On the contrary, if the climatic signal is stronger, the improvement of the quality of series leads to a higher confidence in the detection of the change. It is possible to summarize this outcome by observing that homogenization increases the level of significance/non-significance of the climatic signal.

An important feature of the whole homogenization trial of the precipitation series concerns the correction of snowfall underestimation. The application of this algorithm is also able to correct the attribution of patterns of altitudinal trends in time trends, which, after the application of the correction, become questionable.

The availability of a dense meteorological network in an area offers the important chance to make use of the spatial 
statistical links among series, by working on the spatial coherence criterion. The protocol described in this work still encompasses a wide margin of subjectivity, the acceptance/ rejection of adjustments in series being done manually following suggestions from the software. For example, the presence of a number of suggested change points in the first-level homogenization trial (with no reference), all in the same period for several series, may be rejected, if it is suspected of spotting a period with a true climatic shift. Moreover, an ideal pre-processing of series would encompass a general validation of series, that is, the identification and correction of errors, and not only of the missing data. Such a work would further weigh down the preparatory stage of the climatic analysis. Skipping this task results in definitively rating some series as poorly representative for climatic analysis. In the present work, about $12 \%$ of series were considered non-reliable for climatic analysis even after the whole homogenization trial.

Finally, it is worth to remark the role of climatic series pre-processing (gap filling and homogenization) in the assessment of confidence in past climate trends. The importance of pre-processing has been stated in the reduction of uncertainties in the assessment of mean time trends, with a decrease of standard deviation of trends in the range of $50 \%$. Nevertheless, the trends of homogenized series still show a non-negligible spread. By averaging minimum and maximum temperature trend values, it can be assessed that trends that are inside the 50-year confidence band are far one another up to about $0.12^{\circ} \mathrm{C}$ decade $^{-1}$ (data not shown). So, the difference in mean temperature increase in different sites within the study region can be assessed in as much as $0.60{ }^{\circ} \mathrm{C}$ in 50 years, about one third of the average temperature increase itself (Table 2). Comparatively larger gaps can be assessed for the 30-year series. But, even for a small area like the Trentino region, much larger uncertainty figures should have been commented without a proper preprocessing of original instrumental series.

Acknowledgments This study was carried out in the frame of the projects ACE-SAP and ENVIROCHANGE, funded by the Autonomous Province of Trento and of the PRIN 2008 grant "Water resources assessment and management under climate change scenarios in mountain areas". Thanks to Fondazione E. Mach-CTT (Ufficio GIS), Meteotrentino (PAT), Provincia Autonoma di Bolzano (PAB), ARPA Lombardia, ARPA Veneto for data supply. Thanks to R. De Filippi (Fondazione B. Kessler) and to A. Di Piazza (Fondazione E. Mach) for their suggestions.

\section{References}

Ahrens B (2006) Distance in spatial interpolation of daily rain gauge data. Hydrol Earth Syst Sci 10:197-208

Alexandersson H (1986) A homogeneity test applied to precipitation data. J Climatol 6(6):661-675
Anselmo V (1998) Manuale di riferimento per la misura al suolo delle grandezze idrometeorologiche. CNR-GNDCI, Roma, p 121

Auer I, Boehm R, Jurkovic A, Lipa W, Orlik A, Potzmann R, Schoener W, Ungersboeck M, Matulla C, Briffa K, Jones P, Efthymiadis D, Brunetti M, Nanni T, Maugeri M, Mercalli L, Mestre O, Moisselin J-M, Begert M, Mueller-Westermeier G, Kveton V, Bochnicek O, Stastny P, Lapin M, Szalai S, Szentimrey T, Cegnar T, Dolinar M, Gajic-Capka M, Zaninovic K, Majstorovic Z, Nieplova E (2007) HISTALP-historical instrumental climatological surface time series of the Greater Alpine Region. Int J Climatol 27:17-46

Beniston M, Rebetez M, Giorgi F, Marinucci MR (1994) An analysis of regional climate-change in Switzerland. Theor Appl Climatol 49:135-159

Brugnara Y, Brunetti M, Maugeri M, Nanni T, Simolo C (2011) Highresolution analysis of daily precipitation trends in the central Alps over the last century. Int J Climatol. doi:10.1002/joc.2363

Brunetti M, Maugeri M, Nanni T (2000) Variations of temperature and precipitation in Italy from 1866 to 1995 . Adv Water Resour 65(34):165-174. doi:10.1007/s007040070041

Brunetti M, Lentini G, Maugeri M, Nanni T, Auer I, Bohm R, Schoner W (2009) Climate variability and change in the Greater Alpine Region over the last two centuries based on multi-variable analysis. Int J Climatol 29:2197-2225

Ceppi P, Scherrer SC, Fischer AM, Appenzeller C (2012) Revisiting Swiss temperature trends 1959-2008. Int J Climatol 32:203-213

Ciccarelli N, von Hardenberg J, Provenzale A, Ronchi C, Vargiu A, Pelosini R (2008) Climate variability in north-western Italy during the second half of the 20th century. Glob Planet Chang 63(23): $185-195$

Cohen J, Rind D (1991) The effect of snow cover on the climate. J Clim 4(7):689-706

Della Marta PM (2006) A method of homogenizing the extremes and mean of daily temperature measurements. J Clim 19(17):41794197

R Development Core Team (2011) R: a language and environment for statistical computing. R Foundation for Statistical Computing, Vienna, Austria. ISBN 3-900051-07-0, URL http://www.Rproject.org/

Eccel E, Saibanti S (2007) Inquadramento climatico dell'Altopiano di Lavarone-Vezzena nel contesto generale trentino. Stud Trentini di Sci Nati, Acta Biol 82:111-121

Euskirchen ES, McGuire AD, Chapin FS (2007) Energy feedbacks of northern high-latitude ecosystems to the climate system due to reduced snow cover during 20th century warming. Glob Chang Biol 13(11):2425-2438

Groisman PY, Davies TD (2001) Snow cover and the climate system. In: Jones HG, Pomeroy JW, Walker DA, Hoham RW (eds) Snow ecology. Cambridge Univ Press, New York, pp 1-44

Groisman PY, Karl TR, Knight RW, Stenchikov GL (1994) Changes of snow cover, temperature, and radiative heat balance over the northern hemisphere. J Clim 7(11):1633-1656

Ha-Duong M, Swart R, Bernstein L, Petersen A (2007) Uncertainty management in the IPCC: agreeing to disagree. Glob Environ Chang-Hum Policy Dimens 17(1):8-11

Hantel M, Ehrendorfer M, Haslinger A (2000) Climate sensitivity of snow cover duration in Austria. Int J Climatol 20(6):615-640

Haubner E (2002) I cambiamenti climatici e le Alpi: una relazione specifica. CIPRA, $12 \mathrm{Pp}$. http://www.cipra.org

Jones PD et al (1999) The use of indices to identify changes in climatic extremes. Clim Chang 42(1):131-149

Karl TR, Knight RW, Plummer N (1995) Trends in high frequency climate variability in the 20th century. Nature 377(6546):217-220 
Keller F, Goyette S, Beniston M (2005) Sensitivity analysis of snow cover to climate change scenarios and their impact on plant habitats in Alpine terrain. Clim Chang 72:299-319

Le Treut H, Gastineau G, Li L (2008) Uncertainties attached to global or local climate changes. C R Geosci 340(9-10):584-590

Loffler J (2007) The influence of micro-climate, snow cover, and soil moisture on ecosystem functioning in high mountains. J Geogr Sci 17(1):3-19

Moss RH, Schneider SH (2000) Uncertainties in the IPCC TAR: recommendations to lead authors for more consistent assessment and reporting. IPCC Supporting Material. IPCC, Geneva

Ranzi R, Grossi G, Bacchi B (1999) Ten years of monitoring areal snowpack in the Southern Alps using NOAA-AVHRR imagery, ground measurements and hydrological data. Hydrol Process 13 (12-13):2079-2095

Ranzi R, Grossi G, Gitti A, Taschner S (2010) Energy and mass balance of the Mandrone Glacier (Adamello, Central Alps). Geografia Fisica e Dinamica Quaternaria 33:45-60

Reeves J, Chen J, Wang XLL, Lund R, Lu QQ (2007) A review and comparison of changepoint detection techniques for climate data. J Appl Meteorol Climatol 46(6):900-915

Sevruk B (ed) (1992) Snow cover measurements and areal assessment of precipitation and soil moisture. World Meteorological Organization, operational hydrology report n.35, WMO n.749; Geneva: p. 283

Sevruk B (1996) Adjustment of tipping-bucket precipitation gauge measurements. Atmos Res 42(1-4):237-246

Simolo C, Brunetti M, Maugeri M, Nanni T (2010) Improving estimation of missing values in daily precipitation series by a probability density function-preserving approach. Int J Climatol 30 (10):1564-1576. doi:10.1002/joc.1992

Swart R, Bernstein L, Ha-Duong M, Petersen A (2009) Agreeing to disagree: uncertainty management in assessing climate change, impacts and responses by the IPCC. Clim Chang 92(1-2):1-29
Tegavarapu RSV, Chandramouli V (2005) Improved weighting methods, deterministic and stochastic data-driven models for estimation of missing precipitation records. J Hydrol 312:191-206

Timbal B, Fernandez E, Li Z (2009) Generalization of a statistical downscaling model to provide local climate change projections for Australia. Environ Model Softw 24(3):341-358

Toreti A, Desiato F (2008) Temperature trend over Italy from 1961 to 2004. Theor Appl Climatol 91:51-58

Valt M, Cianfarra P (2010) Recent snow cover variability in the Italian Alps. Cold Reg Sci Technol 64(2):146-157

Wang XLL (2008a) Accounting for autocorrelation in detecting mean shifts in climate data series using the penalized maximal $\mathrm{t}$ or $\mathrm{F}$ test. J Appl Meteorol Climatol 47(9):2423-2444

Wang XLL (2008b) Penalized maximal F test for detecting undocumented mean shift without trend change. J Atmos Ocean Technol 25(3):368-384

Wang XL, Yang F (2007) RHtestV2 user manual. Published online (http://cccma.seos.uvic.ca/ETCCDMI/software.shtml)

Wilby R, Charles S, Zorita E, Timbal B, Whetton P, Mearns L (2004) Guidelines for use of climate scenarios developed from statistical downscaling methods, published online, supporting material to the IPCC, $27 \mathrm{pp}$

WMO-World Meteorological Organisation (2010) CIMO survey on national summaries of methods and instruments for solid precipitation measurement at automatic weather stations, instruments and observing methods report n. 102, Geneva, 2010

Yan ZW, Jones PD (2008) Detecting inhomogeneity in daily climate series using wavelet analysis. Adv Atmos Sci 25(2):157-163

Yang D, Sevruk B, Elomoa E, Golubev V, Goodison B, Gunther T (1994) Wind-induced error on snow measurement: WMO intercomparison results. In: Proc. 23th Int. Conf. Alpine MeteorologyGerman Weather Service, Annalen der Meteorologie, Selbstverlag des Deutschen Wetterdienstes, Offenbach am Main, no. 30:61-64 